\title{
Targeting the Endocannabinoid/CB1
} Receptor System For Treating Major Depression Through Antidepressant Activities of Curcumin and DexanabinolLoaded Solid Lipid Nanoparticles

\author{
Xiaolie He Li Yang Mei Wang Xizhen Zhuang Ruiqi Huang \\ Rongrong Zhu Shilong Wang
}

Research Center for Translational Medicine at East Hospital, School of Life Science and Technology, Tongji University, Shanghai, People's Republic of China

\section{Key Words}

Major depression • Curcumin • Dexanabinol • Solid lipid nanoparticles • Rimonabant • CB1

\begin{abstract}
Background/Aims: This study investigated the underlying mechanisms of the antidepressant effects of curcumin and dexanabinol-loaded solid lipid nanoparticles in corticosterone-induced cell and mice depression models. Methods: Curcumin and dexanabinol-loaded solid lipid nanoparticles (Cur/SLNs-HU-211) were synthesized via an emulsification and low-temperature solidification method. Antidepressant activities of nanoparticles in a corticosterone-induced major depression model were investigated by MTT assay, cellular uptake by flow cytometry, behaviour by Forced Swimming Test and rotarod test, neurotransmitters by High Performance Liquid Chromatography, Western blotting, qPCR and immunofluorescence. Results: Treatment with Cur/SLNs-HU-211 induced greater dopamine (DA)/5-hydroxytryptamine (5-HT) release with reduced corticosterone-induced apoptotic cell death in PC12 cells. Additionally, in vivo Cur/SLNs-HU-211 significantly induced recovery from depressive behaviour with increased DA/5-HT levels, CB1 mRNA levels and CB1, $\mathrm{p}-\mathrm{MEK} 1$ and $\mathrm{p}$-ERK1/2 protein expression levels in the hippocampus and striatum. Cur/SLNs-HU-211 improved CB1 expression and inspired the proliferation of astrocytes in the hippocampus and striatum, exerted neuroprotective effects by preventing corticosterone -induced BDNF/NeuN expression reduction. Conclusion: Our study implies that Cur/SLNs-HU-211 may be a useful approach for treatment of major depression.

(C) 2017 The Author(s)

Published by S. Karger AG, Basel
\end{abstract}

\section{Introduction}

Major depression is a common and highly debilitating disorder that has been ranked by the World Health Organization as a leading cause of disability among oppressive illnesses [1, 2]. Major depression has serious social and economic consequences. Individuals suffering from

Rongrong Zhu,

and Shilong Wang
Research Center for Translational Medicine at East Hospital, School of Life Science and Technology, Tongji University, \#1239 Siping Road, Shanghai 200092 (China)

Tel. +8602165982595, E-Mail rrzhu@tongji.edu.cn / wsl@tongji.edu.cn 
depression endure high risks of complicating comorbidities and reduced quality of life. Despite significant impetus for effective treatments, relatively few promising treatment trials have been reported. No clear explanations for treatment resistance have been reported; however, it is highly possible that current antidepressants do not effectively target all pathological processes in major depression. Thus, there is an urgent need to broaden the targets upon which antidepressants are considered to act.

Curcumin (Cur) is an active component extracted from the roots and stems of plants of the Curcuma species, such as turmeric, radix curcumae, and rhizoma curcumaeis. Cur provides a number of biological activities, including antioxidant and anti-carcinogenic properties and anti-inflammatory and anti-proliferative actions [3-6]. Cur has been found to have antidepressant effects in major depression models, and Cur is believed to display its antidepressant effects through anti-inflammatory, immune-modulating, neuroprotective, mitochondrial, neurotransmitter, hormonal, antioxidative and nitrosative stress activities $[7,8]$. Cur has been regarded as a valuable and promising drug with various advantages for major depression treatment [9]. Dexanabinol (HU-211) is an artificially synthesized cannabinoid derivative that exhibits neuroprotective activities due to its anti-inflammatory and antioxidant effects in the brain. HU-211 could maintain the integrity of the blood brain barrier and decrease cell death by inhibiting NF- $\kappa B$ production and decreasing the expression of cytokines, such as TNF- $\alpha$ and interleukin-6. Different from other cannabinoid derivatives, HU-211 does not act as a cannabinoid receptor agonist and thus, has no effect on the CB1/CB2 receptors, i.e., HU-211 does not induce CB1 expression. Furthermore, HU-211 has been shown to be safe in clinical trials [10] and is now undergoing Phase I trials for the treatment of brain diseases.

Extremely low solubility and poor bioavailability have prevented the uses of Cur and HU-211 as antidepressants. To address these issues, solid lipid nanoparticles (SLNs)-based nanotechnology has been applied. SLNs have several advantages in delivering drugs to the brain, including excellent bioavailability, high drug loading and long circulation time in blood [11]. Considering the efficacy, safety, and non-toxicity of curcumin as an antidepressant and the significant role of HU-211 in brain disease research, we propose the combination of Cur, HU-211 and SLNs to develop Cur/SLNs-HU-211 nanoparticles (NPs) as new curative tools for major depression.

Corticosterone (CORT) has frequently been used in major depression models [12]. CORT has been known to induce PC12 cell apoptosis and depression-like behaviour in mice. Drugs that can attenuate CORT-induced neurotoxicity are believed to have possible therapeutic potential in the treatment of major depression.

The endocannabinoid system is a neuromodulatory system that regulates emotional, cognitive, neurovegetative and motivational processes. Cannabinoid receptor type 1 is a G protein-coupled cannabinoid receptor often found in the central and peripheral nervous system. Evidence has implicated that deficits in endocannabinoids account for the aetiology of major depression [13]. Accordingly, the pharmacological augmentation of endocannabinoid signalling may be a novel target for the pharmacotherapy of major depression [14]. Deficient CB1 receptor (CB1R)-mediated signalling has been reported to result in symptoms that mimic those of major depression. The activation of CB1R-mediated signalling results in behavioural and endocrine effects similar to those generated by currently used antidepressants. Furthermore, recent genetic studies have recognized that individuals with certain CB1R haplotypes have increased vulnerability to depression following adverse life events. Overall, the available data imply that the activators of CB1R signalling should be considered for therapeutic targets for major depression treatment.

Many conventional antidepressant therapies act through enhanced CB1R-mediated signalling. For example, the neuroprotective effects of Cur have been reported to mainly occur through the CBR1-mediated endocannabinoid signalling pathway and the brainderived neurotrophic factor (BDNF) signalling pathway [15]. Based on our previous study, small-sized $60 \mathrm{~nm}$ Cur/SLNs-HU-211 advantageously cross the blood brain barrier and act in the hippocampus and striatum to protect cells from CORT-induced damage (thereby, evoking 
behavioural changes in mice) [16]. We hypothesize that Cur/SLNs-HU-211 nanoparticles (NPs) may have anti-depressant effects on major depression by targeting CBR1. Rimonabant (Rim) as an inverse agonist for CB1R has been used for the treatment of obesity [17]. However, Rim showed greater numbers of anxiety and adverse depression events in clinical trials. In our study, we used Rim to determine whether Cur/SLNs-HU-211 exerted antidepressant effects by targeting CBR1. Additionally, we sought to understand the underlying mechanisms and whether Cur/SLNs-HU-211 treatment could prevent depressive behaviour phenotypes in CORT-treated mice.

\section{Materials and Methods}

\section{Materials}

Curcumin and corticosterone were purchased from Aladdin Chemistry Co., Ltd. (Shanghai, China); rimonabant was purchased from APExBIO Technology (Houston, USA); and HU-211 was purchased from Cayman Chemical (Michigan, USA). All other reagents were of analytical grade. Water used was deionized water.

\section{Fabrication of nanoparticles}

Cur/SLNs-HU-211 were synthesized using an emulsification and low-temperature solidification method. Briefly, $10 \mathrm{~mL}$ chloroform containing Cur (0.15 g), stearic acid (0.2 g), lecithin (0.1 g), and HU$211\left(0.002 \mathrm{~g}\right.$ ) were added to $30 \mathrm{~mL}$ of $\mathrm{H}_{2} \mathrm{O}$ containing polyoxyethylene stearate $(0.25 \mathrm{~g})$ under $1,200 \mathrm{rpm}$ stirring at $75^{\circ} \mathrm{C}$. Then, $10 \mathrm{~mL}$ of $\mathrm{H}_{2} \mathrm{O}$ was added to bring the total solution volume to $15 \mathrm{~mL}$. The solution was stirred for $2 \mathrm{~h}$ at $4^{\circ} \mathrm{C}$. The final product was dried at $-56^{\circ} \mathrm{C}$ under vacuum.

\section{Characterization of nanoparticles}

The morphology of the nanoparticles was observed via transmission electron microscopy (JEOL, Tokyo, Japan). Nanoparticle zeta potential was measured using a Malvern zetasizer Nano ZS (Malvern Instrument, UK). The drug loadings of Cur and HU-211 in Cur/SLNs-HU-211 were measured with ultraviolet-visible spectroscopy (UV-Vis) and High Performance Liquid Chromatography (HPLC), respectively.

Cell culture and animals

PC12 cells were cultured according to a previously reported method. Dulbecco's Modified Eagle's Medium containing penicillin (100 unit/mL), streptomycin $(100 \mu \mathrm{g} / \mathrm{mL}), 5 \%$ foetal bovine serum, and $10 \%$ horse serum were used. PC12 cells were placed in a $5 \% \mathrm{CO}_{2}$ humidified incubator at $37^{\circ} \mathrm{C}$.

C57BL/ 6 mice were purchased from the Laboratory Animal Centre of Tongji University. Animal protocols were approved by the institutional research ethics committee of Tongji University and in accordance with the National Institutes of Health Guide for the Care and Use of Laboratory Animals.

Analysis of DA/5-HT level release from PC12 cells

To compare the antidepressant effects of Cur, HU-211, Cur+HU-211, Cur/SLNs-HU-211 (at a Cur dose of $1 \mu \mathrm{M}$ ), 5-HT level in vitro were measured via HPLC. Cells were grouped as Control, CORT, CORT+ Cur, CORT+ HU-211, CORT+Cur+ HU-211, and CORT+Cur/SLNs-HU-211 nanoparticles. For 5-HT, the mobile phase consisted of methanol/0.05 $\mathrm{M} \mathrm{CH}_{3} \mathrm{COONa}(60: 40, \mathrm{v} / \mathrm{v})$ at a $0.5 \mathrm{~mL} / \mathrm{min}$ flow rate, and a $275 \mathrm{~nm}$ detection wavelength.

To investigate the antidepressant effects of Cur/SLNs-HU-211 and the inhibition effect of Rim, the release of DA/5-HT from PC12 cells was detected. The cells were seeded in a 12-well plate in five groups: Control, Rim $(10 \mu \mathrm{M})$, Corticosterone (CORT) $(300 \mu \mathrm{M})$ as the major depression model group, CORT (300 $\mu \mathrm{M})+\mathrm{NPs}(1 \mu \mathrm{M}$ Cur $)$, and CORT $(300 \mu \mathrm{M})+\mathrm{NPs}(1 \mu \mathrm{M}$ Cur $)+\operatorname{Rim}(10 \mu \mathrm{M})$. Culture media were collected after various treatments for $24 \mathrm{~h}$. To determine the DA/5-HT level in the media, an HPLC instrument was used. For DA, separation was conducted with a mobile phase consisting of methanol/0.01 $\mathrm{M} \mathrm{KH}_{2} \mathrm{PO}_{4}(10: 90$, $\mathrm{v} / \mathrm{v}, \mathrm{pH} 3.5$ ) at $0.5 \mathrm{~mL} / \mathrm{min}$ and a detection wavelength of $280 \mathrm{~nm}$. For 5 -HT, the settings were as described above. 


\section{Cellular Physiology Cell Physiol Biochem 2017;42:2281-2294

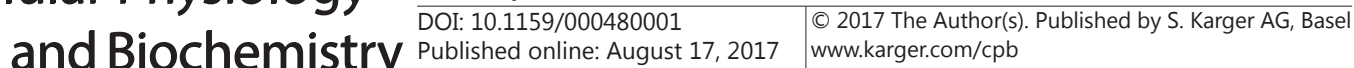 \\ He et al.: Antidepressant Effects of Cur/SLNs-HU-211 Exerted by Targeting CB1R in Major}

Depression

Standard DA/5-HT samples were used to obtain a standard curve, and the concentrations of DA/5-HT in the media were determined from the standard curve.

\section{MTT assay}

To compare the antidepressant effects of Cur, HU-211, Cur+HU-211, Cur/SLNs-HU-211 (at a Cur dose of $1 \mu \mathrm{M}$ ), the MTT assay was used. PC12 cells were seeded in a 96-well plate. The division of groups was the same as mentioned before. $24 \mathrm{~h}$ after treatment, cell medium was refreshed with $0.5 \mathrm{mg} / \mathrm{mL}$ MTT, and the cells were cultured for an additional $4 \mathrm{~h}$ at $37^{\circ} \mathrm{C}$. DMSO was then added to the wells, which were shaked for approximately $10 \mathrm{~min}$. Absorbance at $492 \mathrm{~nm}$ was measured. The results are presented as percentages of the control.

To investigate the antidepressant effects of Cur/SLNs-HU-211 and the inhibition effects of Rim, the MTT assay was also used.

\section{Western blot in vitro}

Cells were seeded in a 6-well plate and given various treatments. After the treatments, PC12 cells were collected and washed with phosphate buffer saline (PBS). Total proteins were extracted, separated and transferred onto a PVDF membrane. The membrane was blocked with $5 \%(\mathrm{w} / \mathrm{v})$ bovine serum albumin (BSA) in TBST for $1 \mathrm{~h}$ and incubated with primary antibodies (CB1, p-MEK1, and p-ERK1/2) overnight at $4^{\circ} \mathrm{C}$. After washing with TBST, the membrane was incubated with second antibodies for $1 \mathrm{~h}$ at room temperature. Finally, bands were observed using a chemiluminescence detection system (Santa Cruz Biotechnology, Santa Cruz, CA, USA).

\section{Cellular uptake detection}

To analyse whether Rim will suppress the cellular uptake behaviour of Cur/SLNs-HU-211, PC12 cells were seeded in a 6-well plate for $24 \mathrm{~h}$. Then, the media were exchanged with culture media with different treatment agents (CORT + Cur/SLNs-HU-211 NPs and CORT + Cur/SLNs-HU-211 NPs + Rim). The cells were treated for 1, 2, 4, and $8 \mathrm{~h}$. Afterward, the cells were washed three times with PBS, collected, and washed twice to detect Cur at $488 \mathrm{~nm}$ with a flow cytometer.

\section{Drug distribution in vivo}

Normal mice were separated into three group and given Cur, HU-211 or Cur/SLNs-HU-211 (at a Cur dose of $20 \mathrm{mg} / \mathrm{kg}$ ). Mice brains were sampled at predetermined time points (5, 15, 30, 45, 60, 120, 240, and $360 \mathrm{~min}$ ). HPLC was used to determine drug concentrations in the brain, and the protein concentrations of the tissue were immediately quantified.

\section{CORT-induced major depression mice model}

A major depression mice model was established under repeated daily administration of CORT (40 mg/ $\mathrm{kg}$ ) for 3 weeks. Then, mice were randomly assigned to five groups: Group I, normal control mice; Group II, normal control mice treated with Rim (3 mg/kg); Group III, mice with major depression that received PBS; Group IV, mice with major depression that were subjected to Cur/SLNs-HU-211(at a Cur dose of $20 \mathrm{mg} / \mathrm{kg}$ ); and Group V, mice with major depression that received Cur/SLNs-HU-211 and Rim (3 mg/kg). Drugs were given daily.

\section{Behavioural change observation}

A Forced Swimming Test (FST) was carried out to measure behavioural changes after drug treatment. Each mouse was placed in a glass cylinder filled with water for $8 \mathrm{~min}$, and movement was recorded by camera. Because immobility was not observed in the first $2 \mathrm{~min}$, the final $6 \mathrm{~min}$ were measured by a blinded scorer. Mice were considered immobile when they stopped struggling in the water.

One day after the mice finished the FST, the same mice underwent a rotarod test. The apparatus comprised a drum that rotated at a speed of $4 \mathrm{rpm}$, which slowly increased to $40 \mathrm{rpm}$ within $5 \mathrm{~min}$. The experiment was measured by counting the latency of mice staying on the drum.

\section{Brain neurotransmitters analysis}

Brain regions, including the cortex, hippocampus, brainstem, cerebellum, and striatum, were obtained and homogenized for neurotransmitter measurement. DA/5-HT levels were measured as previously 
mentioned. The protein concentrations of each region were quantified and the results are reported as the concentrations of DA/5-HT per mg protein.

Table 1. Primer sequences for real-time PCR of target genes

\begin{tabular}{ll}
\hline Primer name & Primer sequence \\
\hline GAPDH & Forward Primer: 5' -AGGTCGGTGTGAACGGATTTG - 3' \\
& Reverse Primer: 5' -TGTAGACCATGTAGTTGAGGTCA - 3' \\
CB1 & Forward Primer: 5' -TCAAGGAGAACGAGGACAACA- 3' \\
& Reverse Primer: 5' -CCAGGGTGAGGGACAGGA-3' \\
\hline
\end{tabular}

\section{$q P C R$}

The total RNA of the cortex, hippocampus, brainstem, cerebellum, and striatum were isolated using Trizol reagent (Invitrogen). The concentration and purity of RNA samples were detected by a Nanodrop ND-2000 (Thermo Science, USA). cDNA was synthesized with a primer Script Reverse Transcriptase Kit (Takara). Quantitative realtime PCR was performed using SYBR Premix Ex Taq $^{\text {TM }}$ (Takara) on QuantStudio 7 Flex Real-Time PCR System. The primer sequences (Sangon Biotech, China) are listed in Table 1. Relative amounts of mRNA were calculated by the $\Delta \Delta \mathrm{Ct}$ relative quantification method. GAPDH served as the control gene and the mRNA levels of specific genes were normalized to GAPDH.

Western blot

The total proteins in the hippocampus and striatum cells were extracted. Primary antibodies CB1 (ab23703, abcam), MEK1 (ab32091, abcam), p-MEK1 (ab214445, abcam), ERK1/2 (ab184699, abcam), p-ERK1/2 (ab32538, abcam) and $\beta$-actin (ab8227, abcam) were used. The Western blot procedure is described above.

\section{Immunofluorescence}

Briefly, 15- $\mu \mathrm{m}$-thick sections were incubated with a blocking buffer (1× PBS/5\% normal goat serum $/ 0.3 \%$ Triton X-100) for $1 \mathrm{~h}$. The sections were incubated with the following primary antibodies overnight in PBS at 1:500 dilution factors at $4^{\circ} \mathrm{C}$ : CB1 (ab23703, abcam) were conjugated with $\mathrm{CF}^{\mathrm{TM}} 405 \mathrm{~S}$ Dye (Biotium, Shanghai), glial fibrillary acidic protein (GFAP, ab49874, abcam, conjugated with Cy3), neuronal nuclei (NeuN, ab190195, abcam, conjugated with Alexa Fluor 488) and BDNF (bs-4989R-A647, Bioss, conjugated with Alexa Fluor 647). After washing with PBS, RedDot (Biotium, Shanghai) and DAPI were used for nuclei staining. Later, the sections were observed under a Leica (Wetzlar, Germany) TCS SP5 confocal microscope.

\section{Statistical analysis}

All results are presented as means \pm standard deviation (SD). Statistical analyses were performed using the Statistical Product and Service Solutions software. One-way analysis of variance was performed for each analysis. Significance was set at $P$ values less than 0.05 . Semi-quantitative analyses were conducted using ImagePro Plus software.

\section{Results}

\section{Characterization of NPS}

Cur/SLNs-HU-211 NPs were successfully prepared. The NPs were characterized by transmission electron microscopy (TEM). As seen from Fig. 1A, Cur/SLNs-HU-211 had spherical shapes and uniform size. The zeta potential distribution (Fig. 1B) showed that Cur/SLNs-HU-211 ( $-22.6 \pm 0.9 \mathrm{mV})$ exhibited a negative zeta potential. The Cur and HU-211 loading efficiencies in Cur/SLNs-HU-211 as analysed by UV-Vis spectrophotometry and HPLC were $19.12 \% \pm 1.43 \%$ and $0.81 \% \pm 0.04 \%$, respectively. 


\section{Cur/SLNs-HU-211 NPS showed betterantidepressant effects}

To determine whether Cur/ SLNs-HU-211 showed greater antidepressant effects in vivo, 5-HT release and MTT assays were conducted. As shown in Fig. 2A, Cur, HU-211 and Cur + HU211 showed limited increases in 5-HT level. However, Cur/SLNsHU-211 significantly improved 5-HT release from cells when compared with Cur+HU-211. In the MTT assay (Fig. 2B), both Cur and HU-211 protected cells from CORT-induced cell damage and death. Furthermore, Cur/SLNs-HU-211 showed stronger protective effects than Cur+HU-211.

Rim attenuate the protective effects of Cur/SLNs-HU-211 NPs on major depression cell model

DA/5-HT release was measured to investigate the effects of Cur/SLNs-HU-211 in a major depression model in PC12 cells. As seen in Fig. 3A and Fig. $3 \mathrm{~B}$, in the presence of CORT, Cur/ SLNs-HU-211 NPs up-regulated DA/5-HT levels, which indicated that Cur/SLNs-HU-211 has the potential to protect cells from CORT. However, this effect was weakened when Rim was added.

MTT assays were performed to evaluate the effect of Cur/ SLNs-HU-211 on CORT induced apoptosis in PC12 cells. In the assay, the cell viabilities of different groups (i.e., Control, Rim, CORT, CORT + NPs and CORT + NPs + Rim) were 100\%, 75.4\%,

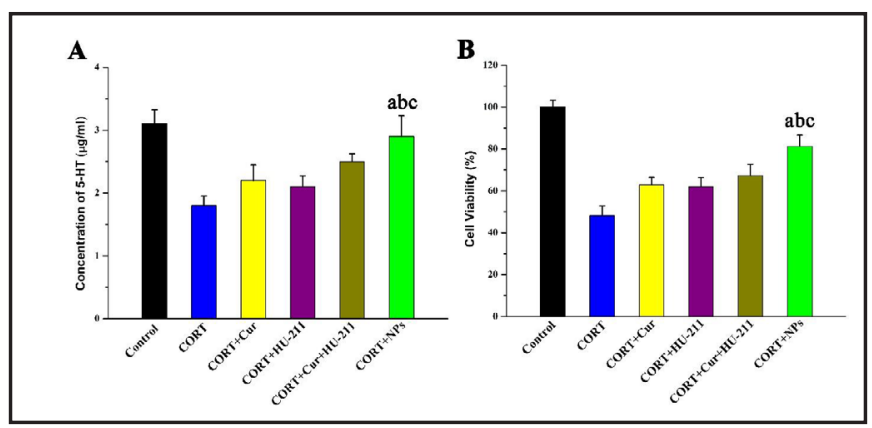

Fig. 2. Comparison of different treatments in PC12 cell (A) 5-HT level for different treatments in PC12 cells. (B) Cells viability determined by MTT assay. For significance, ${ }^{\mathrm{P}}<0.05$ compared with CORT+Cur group, ${ }^{b} \mathrm{P}<0.05$ compared with CORT + HU-211 group, ${ }^{c} \mathrm{P}<0.05$ compared with CORT $+\mathrm{Cur}+\mathrm{HU}-211$ group.

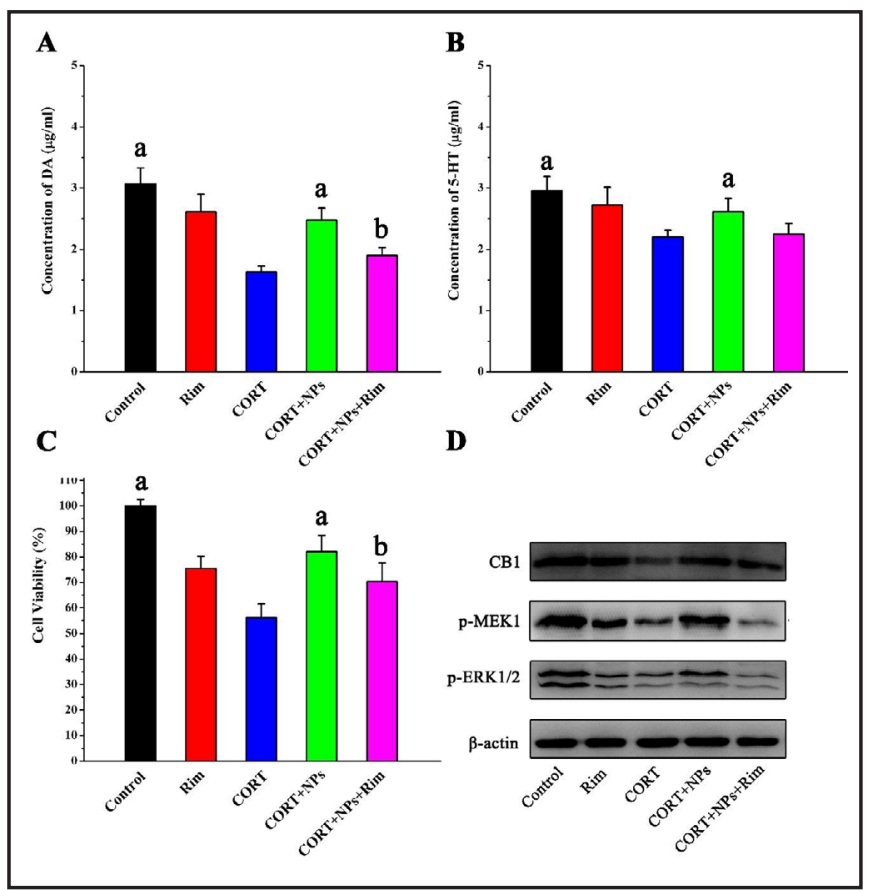

Fig. 3. Protective effects of Cur/SLNs-HU-211 on PC12 cell (A) DA level for different treatments in PC12 cells. (B) 5-HT release from PC12 cells. (C) Cells viability determined by MTT assay. (D) Western blot for CB1, p-MEK1, and p-ER K1/2 protein in vitro. For significance, ${ }^{\mathrm{a}} \mathrm{P}<0.05$ compared with CORT group; ${ }^{\mathrm{b}} \mathrm{P}<0.05$ compared with CORT+NPs group.

$56.1 \%, 81.9 \%$ and $70.3 \%$, respectively (Fig. 3C), suggesting that Cur/SLNs-HU-211 protected cells from CORT-induced cell apoptosis, whereas Rim had the opposite effect.

The results of the DA/5-HT release and MTT assays show that Cur/SLNs-HU-211 had great potential in protecting PC12 cells from CORT-induced damage. To further understand the underlying mechanism behind this protective effect, Western blot analysis was carried out. We detected CB1, p-MEK1, and p-ERK1/2 protein expression in all groups. These expression levels were markedly lower in the CORT-treated group when compared with those of the normal control. Their activities were increased with Cur/SLNs-HU-211 treatment. Rim 
treatment significantly downregulated the expression levels in the presence of NPs (Fig. 3D).

As seen from the cellular uptake results (Fig. 4), cells treated with Cur/SLNs-HU-211 exhibited much stronger fluorescence intensities when compared with Rim at different time points. Approximately $79.7 \%$ of total cells were detected after $8 \mathrm{~h}$ treatment with Cur/SLNs-HU-211 and only $52.1 \%$ with Rim. Cellular uptake in PC12 cells was time

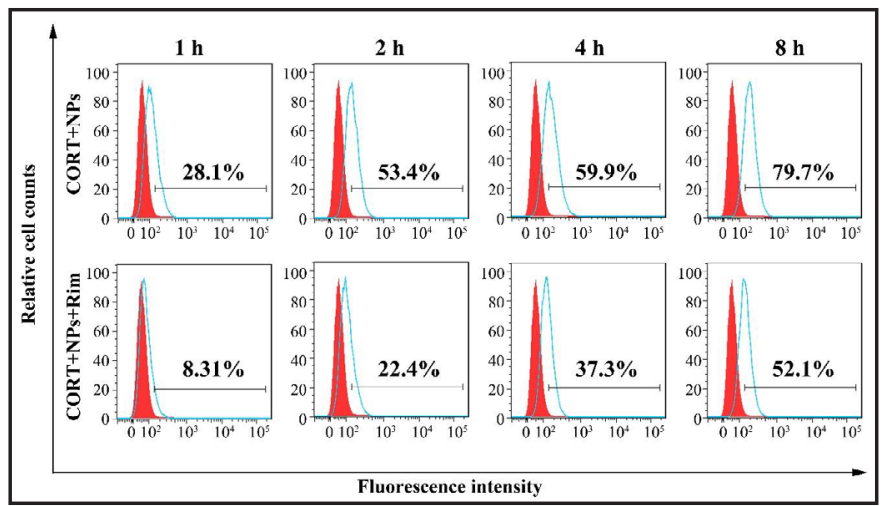

Fig. 4. Cellular uptake observation of Cur/SLNs-HU-211 in PC12 cells by flow cytometry at $1,2,4$, and $8 \mathrm{~h}$. dependent for each group. Cur/SLNs-HU-211 were easily taken up by PC12 cells. Rim suppressed this effect, which suggested that Rim may attenuate the Cur/ SLNs-HU-211-induced antidepressant effects through decreased cellular uptake.

Cur/SLNs-HU-211 improved drug distribution in vivo

Drug distribution was measured using HPLC (Fig. 5). For the HU-211-treated group, no HU-211 was observed in brain samples because the HU-211 in the Cur/SLNs-HU-211 nanoparticles were too low to measure. For the Cur-treated group, Cur can be clearly found at a

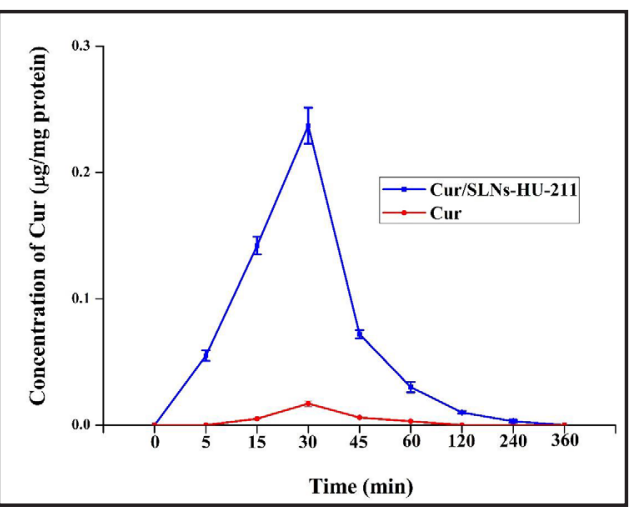

Fig. 5. In vivo drug distribution for several time points $(5,15,30,45,60,120,240,360 \mathrm{~min})$. few time points in the curve. A peak appeared at approximately $30 \mathrm{~min}$ after the drug was given, and Cur did not remain in the body for a long time. However, when compared with the Cur-treated group, Cur/SLNs-HU-211 significantly improved the distribution of Cur in the brain and the bioavailability of Cur in vivo. Furthermore, Cur/SLNs-HU-211 achieved a sustained release of Cur for approximately $6 \mathrm{~h}$ in vivo.

Rim attenuate the Cur/SLNs-HU-211 NPs induced behavioural recovery and neurotransmitters levels rise

The schematic shown in Fig. $6 \mathrm{~A}$ shows that the experiments were conducted using a major depression mice model. We first established a CORT-induced major depression mice model in the first 3 weeks [18]. Then, we treated mice with different drugs for the following 2 weeks. Subsequently, behavioural changes were observed. We then harvested the tissue samples for DA/5-HT analysis.

To determine whether Cur/SLNs-HU-211 suppressed depressive behaviours in the CORT-treated mice, we assessed multiple dimensions of depressive behavioural phenotypes. The FST and rotarod tests are two reliable and frequently used methods for evaluating behavioural changes in mice [19-22].

The results of the FST (Fig. 6B) revealed that mice with major depression (78 seconds) showed significant decreases in float duration when compared with Control mice (126 seconds). Cur/SLNs-HU-211-treated mice significantly increased the float time (109 seconds) when compared with CORT (78 seconds). Rim attenuated the effect of Cur/SLNs-HU-211 by significantly decreasing the float time ( 45 seconds). The results of the rotarod test (Fig. 6C) also showed that Cur/SLNs-HU-211 (288 seconds) significantly enhanced fall latency when 


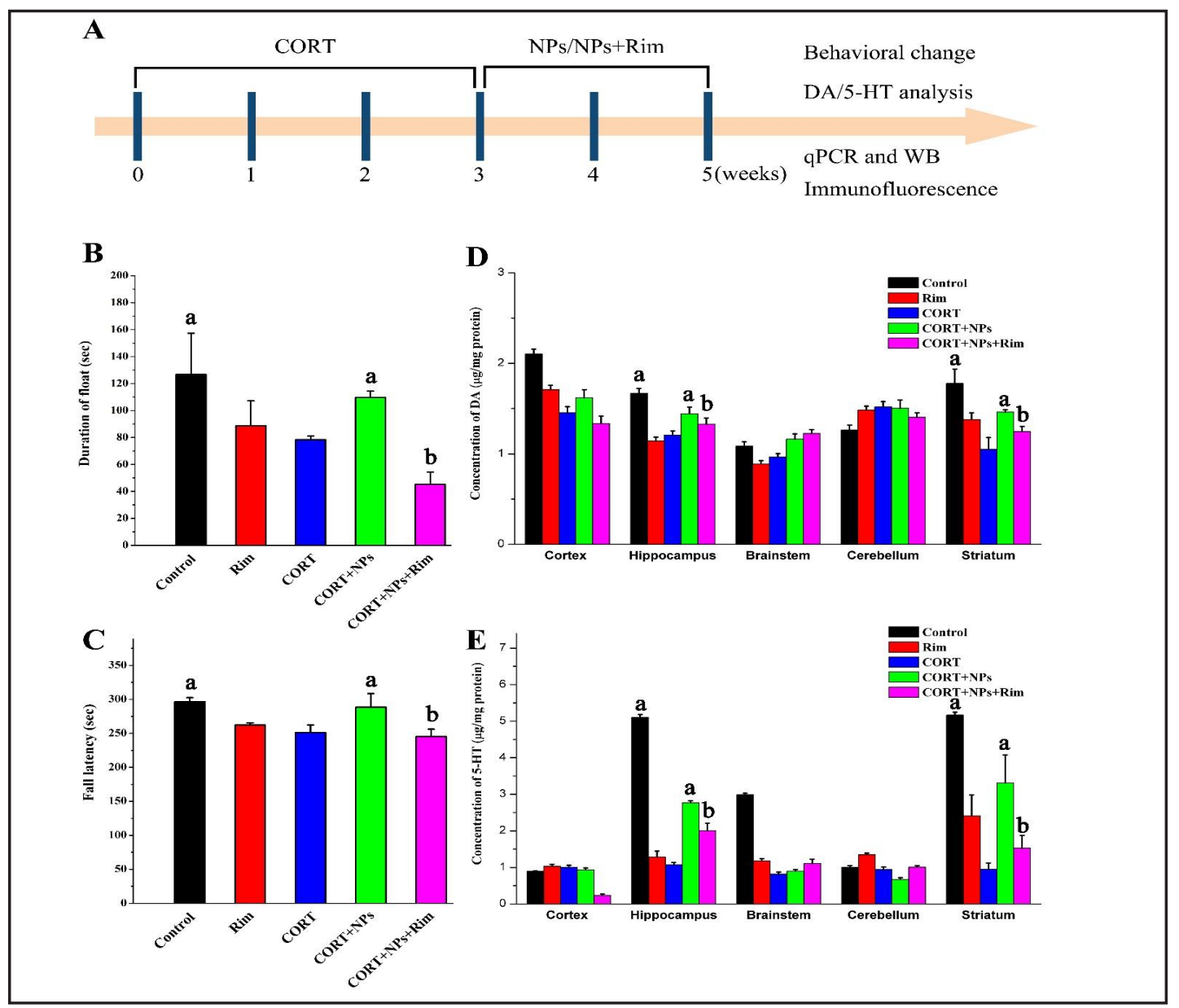

Fig. 6. Antidepressant activities of Cur/SLNs-HU-211 on mice depression model. (A) Schematic shows the establishment of CORT-induced major depression mice model, the drug given and different evaluation; (B) Duration of float in the forced swim test; (C) Fall latency in the rotarod test; (D) Measurement of DA in cortex, hippocampus, brainstem, cerebellum, and striatum; (E) Detection of 5-HT in cortex, hippocampus, brainstem, cerebellum, and striatum. For significance, ${ }^{a} \mathrm{P}<0.05$ compared with CORT group; ${ }^{\text {b }}<0.05$ compared with CORT+NPs group.

compared with CORT (247 seconds). In the Rim + CORT + Cur/SLNs-HU-211 group, Rim (245 seconds) decreased the fall latency induced by Cur/SLNs-HU-211.

We also measured the levels of DA/5HT in the cortex, hippocampus, brainstem, cerebellum, and striatum of mice brains. The results (Fig. 6D and Fig. 6E) showed that Cur/SLNs-HU-211 significantly induced more DA/5-HT compared with the CORT group especially in the hippocampus and striatum. Furthermore, the concentration of DA/5-HT in the Cur/SLNs-HU-211 + Rimtreated group was lower than that of the Cur/SLNs-HU-211-treated group. Overall, these etiology and biochemical marker

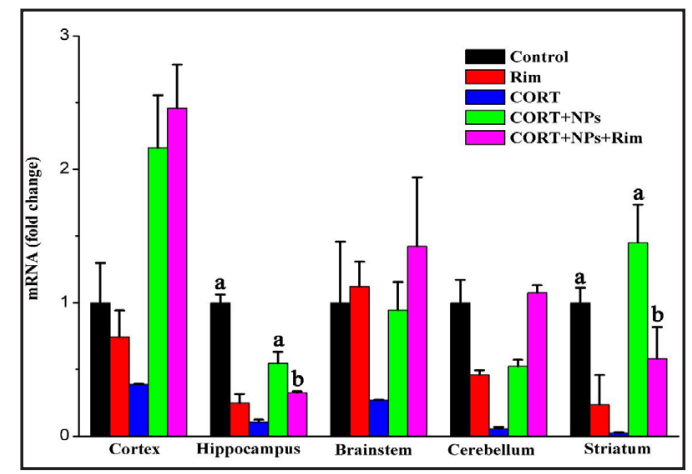

Fig. 7. Protective effects of Cur/SLNs-HU-211 on mice depression model. qPCR assay for the expression of CB1 in brain region; For significance, ${ }^{\mathrm{a}} \mathrm{P}<0.05$ compared with CORT group; ${ }^{\mathrm{b}} \mathrm{P}<0.05$ compared with CORT+NPs group. 
Fig. 8. Protective effects of Cur/SLNsHU-211 on mice depression model. (A) Western blot images for CB1, MEK1, p-MEK1, ERK and $\mathrm{p}$-ERK1/2 for hippocampus and semiquantitate for protein expression level compared with Control; (B) Western blot images for CB1, MEK1, p-MEK1, ERK and p-ERK1/2 for striatum and semiquantitate for protein expression level compared with Control. For significance, $\quad{ }^{a} \mathrm{P}<0.05$ compared with CORT group; ${ }^{b} \mathrm{P}<0.05$ compared with CORT+NPs group.

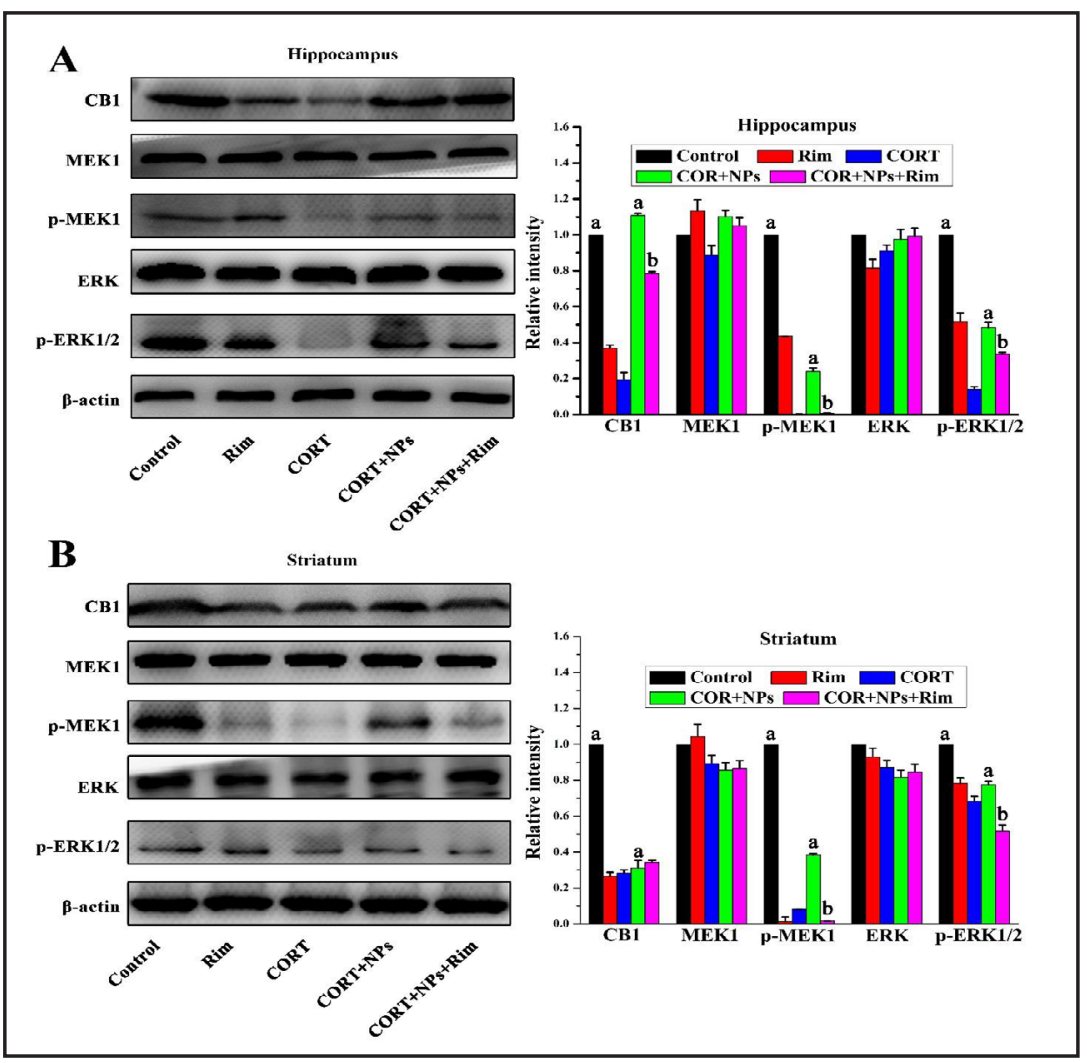

measurements implied that Cur/SLNs-HU-211 showed curative effects in improving the mobility and DA/5-HT levels in vivo. Furthermore, Rim can inhibit these Cur/SLNs-HU-211induced changes.

Rim supressed the Cur/SLNs-HU-211 NPs induced MEK1/ERK1/2 signalling activation

The results described above encouraged us to further investigate the antidepressant activity of Cur/SLNs-HU-211 at the mRNA and protein levels. CB1 mRNA expression levels in the cortex, hippocampus, brainstem, cerebellum, and striatum were measured. The results (Fig. 7) suggested that in the hippocampus and striatum, the CB1 mRNA levels decreased when the rats were treated with CORT. The addition of Cur/SLNs-HU-211 increased the CB1 mRNA levels. In the CORT + NPs + Rim group, Rim treatment showed the opposite effect when compared with the Cur/SLNs-HU-211 group because the CB1 mRNA level significantly decreased.

CB1 cannabinoid receptors have been reported to be vital in alleviating major depression. The activation of CB1 activates the downstream signalling molecules ERK1/2, which occurs via an upstream MEK1 response. Thus, we further investigated the CB1, p-MEK1 and p-ERK1/2 protein expression levels in the hippocampus and striatum. The results (Fig. 8A and Fig. 8B) revealed that for the CORT-treated mice, the CB1, p-MEK1 and p-ERK1/2 protein expression levels were significantly decreased in the hippocampus and striatum. However, Cur/SLNs-HU-211 treatment improved the protein levels; adding Rim inhibited the protein expression.

Rim supressed the Cur/SLNs-HU-211 NPs induced astrocytes activation and BDNF/NeuN expression increase

To confirm the results of the qPCR and Western blot assays, immunofluorescent imaging of mice brain samples (hippocampus and striatum) were performed. CB1 expression and astrocytes activation were most prominent in the hippocampus and striatum of the CORTinduced major depression mice [23, 24]. 
Fig. 9. Representative immunofluorescence images for CB1, GFAP and RedDot in hippocampus and striatum after treatment. (A) Immunofluorescence images for hippocampus; (B) semi-quantitate for CB1 positive cells in hippocampus; (C) semi-quantitate for GFAP positive cells in hippocampus; (D) Immunofluorescence images for striatum; (E) semi-quantitate for CB1 positive cells in striatum; (F) semi-quantitate for GFAP positive cells in striatum; ${ }^{\mathrm{a}} \mathrm{P}<0.05$ compared with CORT group; ${ }^{b} \mathrm{P}<0.05$ compared with CORT+NPs group.

In the hippocampus, to examine the effect of Cur/SLNsHU-211 on CB1 expression, we employed CF Dye to conjugate with CB1. As shown in Fig. 9A and Fig. 9B, when compared with the Control, CORT induced significant decreases in CB1 expression. However, greater CB1 expression was observed when the mice were treated with Cur/SLNs-HU-211. Rim treatment showed the opposite effect induced by Cur/SLNsHU-211, which indicated that the protective function of Cur/ SLNs-HU-211 on major depression mice was largely due to CB1 receptor activation. We used the known GFAP marker for astrocytes to assess the effects of Cur/SLNs-HU-211 on astrocyte activation (Fig. 9A and Fig. 9C). The CORT-treated group showed decreased GFAP expression when compared with the Control. Treatment with Cur/SLNs-HU-211 induced more GFAP-positive astrocytes, which implied that Cur/ SLNs-HU-211 may play important roles in neurogenesis and major depression treatment.

The results from the striatum were similar to those observed in the hippocampus. As shown in Fig. 9D and Fig. 9E, for CB1 expression, Cur/SLNs-HU-211 protected the striatum from CORT-induced CB1 expression decrease, and Rim inhibited this function. For GFAP expression, CORT-induced astrocyte loss was restored with Cur/SLNs-HU-211 treatment, and Rim decreased GFAP expression in the presence of Cur/SLNs-HU-211.

BDNF also regulates neurogenesis, proliferation, and neuronal survival [25-27]. BDNF/ NeuN expression levels were markedly decreased in the hippocampus and striatum of the
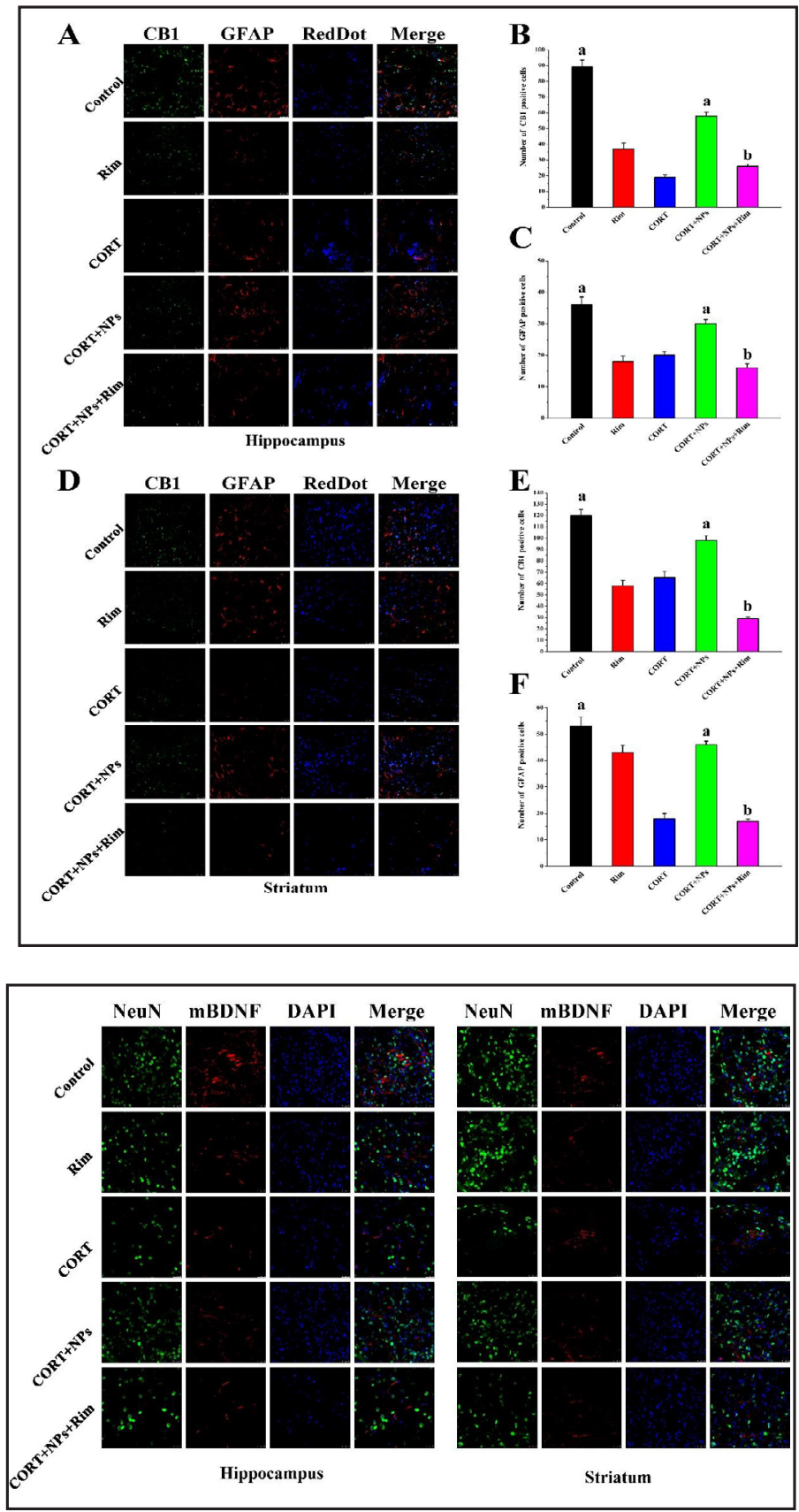

Fig. 10. Representative immunofluorescence images of NeuN and BDNF expression in hippocampus and striatum. 
Rim/CORT-treated mice compared with those of the Control (Fig. 10). However, BDNF/NeuN expression levels significantly recovered in Cur/SLNs-HU-211-treated mice, suggesting that Cur/SLNs-HU-211 may induce neuroprotective effects on CORT-treated mice via the upregulation of BDNF and NeuN expression. In the CORT + NPs + Rim group, Rim attenuated the Cur/SLNs-HU-211 NP-induced increase in BDNF/NeuN expression. This revealed that Cur/SLNs-HU-211 had antagonistic effects.

Overall, these immunofluorescence data implied that Cur/SLNs-HU-211 could be an effective drug for neuronal injury recovery and that Rim could influence this therapeutic effect.

\section{Discussion}

Cur is a traditional herbal medicine that has been used to cure major depression due to its neuroprotective effects. HU-211 shows antidepressant properties due to its antiinflammatory and antioxidant effects. However, because of the low solubility and poor bioavailability of Cur and HU-211, we improved their antidepressant effects with the help of nanoparticles. In this study, we combined SLNs with Cur and HU-211 to synthesize a new dual-drug nanoparticle (Cur/SLNs-HU-211) for enhanced antidepressant effects.

Cur/SLNs-HU-211 nanoparticles were prepared via an emulsification and lowtemperature solidification method. The resultant uniform sizes of the Cur/SLNs-HU-211 enable better solubility and bioavailability. A negative potential of $-22.6 \mathrm{mV}$ provided increased stability and prevented aggregation. These characterization data implied that the fabrication of these nanoparticles was feasible. These nanoparticles enhance the efficacy and delivery of these drugs.

To prove that our synthesized nanoparticles had enhanced antidepressant activities, in vitro 5-HT release and MTT assays were used in a CORT-induced major depression PC12 cells model. The Cur/SLNs-HU-211 were superior to Cur, HU-211 or Cur + HU-211.

We evaluated the in vitro beneficial effects of Cur/SLNs-HU-211 on depressive behaviours to determine the underlying mechanisms in a cell model of major depression. A cannabinoid receptor CB1 inverse agonist Rim was used to show that Cur/SLNs-HU-211 exerted its antidepressant effects by targeting the CB1 receptor. As is well-known, neurotransmitters such as DA/5-HT are vital in the pathogenesis of major depression. Nearly all antidepressant strategies have been shown to enhance 5-HT transmission in the brain of laboratory animals. DA can also play a pivotal role in the mechanism of action of certain antidepressant strategies. Treatment with Cur/SLNs-HU-211 induced more DA/5-HT release with a reduction of CORTinduced apoptotic cell death in PC12 cells. Cur/SLNs-HU-211 as an antidepressant can improve the level of neurotransmitters and protect cells from apoptosis, which is beneficial in the treatment of major depression.

The in vitro mechanism was explored using Western blots and cellular uptake analyses. The Western blot assays implied that Cur/SLNs-HU-211 may activate the CB1 receptor and affect the MEK1/ERK1/2 signalling pathway. Rim significantly inhibited Cur/SLNs-HU-211 uptake by PC12 cells, which showed that Rim may weaken Cur/SLNs-HU-211-induced CB1 receptor activation.

Cur/SLNs-HU-211 increased the in vivo distribution of Cur in mice brains and maintained drug release for prolonged periods, which facilitates drug delivery and antidepressant effects in biological systems. Antidepressants can prevent repeated CORT-induced depression-like behaviour in mice. The Forced Swimming Test and the rotarod test are two frequently applied behavioural tests for major depression research. Cur/SLNs-HU-211 treatment induced recovery from depressive behaviours in the Forced Swimming Test and the rotarod test $[28,29]$. Rim reversed these improvements. These results suggest that Cur/SLNs-HU-211 enabled the mice to maintain motor activity and coordination.

Various studies have suggested that the pathogenesis of major depression is closely correct with the hippocampus and striatum in the brain region. Thus, 5-HT/DA levels in the hippocampus and striatum are important and serve as biomarkers reflecting major 
depression. Cur/SLNs-HU-211 displayed protective effects by elevating neurotransmitter (5-HT/DA) levels in mice brain, especially in the hippocampus and striatum.

The pharmacological augmentation of endocannabinoid signalling could be a target for the treatment of major depression. CB1R signalling is an important therapeutic target for major depression and has attracted widespread attention. CB1 is closely related with the MEK1/ERK1/2 signalling pathway. ERK1/2 is widely retained in mammalian nervous system and is indispensable for the functions of normal brain and major depression. The activation of ERK1/2 occurs through an upstream MEK1 response. Previous studies have implied that acute tetrahydrocannabinol administration could elevate CB1 receptor-mediated ERK1/2 activation in the striatum and hippocampus. Furthermore, Cur can activate the MEK1/ ERK1/2 signalling pathways through CB1 cannabinoid receptors. Our data implied that Cur/SLNs-HU-211 was neuroprotective by enhancing the expression of CB1 and activating MEK1/ERK1/2 signalling. Cur/SLNs-HU-211 raised the mRNA level of CB1 and the protein expression levels of CB1, p-MEK1 and p-ERK1/2 in the hippocampus and striatum. However, Rim, as an inverse agonist for CB1R, reduced the activation of p-MEK1, p-ERK1/2 in the presence of Cur/SLNs-HU-211, further suggesting that Cur/SLNs-HU-211 was involved in MEK1/ERK1/2 signalling.

Reductions in astrocytes and related markers (GFAP) have been considered important characteristics in the pathology of major depression. Astrocytes are the most numerous and versatile of all types of glial cells. They are crucial to neuronal microenvironments and regulate glucose metabolism, neurotransmitter uptake synaptic development and maturation. CORT induces astrocyte loss in the striatum and hippocampus, resulting in the prominent pathophysiological development of major depression. This was confirmed in our immunofluorescence assays. This phenomenon was rescued by Cur/SLNs-HU-211.

BDNF is believed to be a key modulator of neurogenesis and contributes to cell proliferation and neuronal survival. Cur/SLNs-HU-211 restored BDNF/NeuN reductions induced by CORT. This implied that Cur/SLNs-HU-211 may ameliorate neurodegenerative symptoms and enhance the recovery of neuronal function. Current theories for major depression have focused on the hypothalamus-pituitary-adrenal axis, neurotrophin signalling and neuroinflammation. However, the correlation between neuroprotection and enhanced neurogenesis and behavioural recovery from depressive phenotypes by Cur/SLNs-HU-211 suggests the effectiveness of Cur/SLNsHU-211 treatment for major depression via the attenuation of neuronal injury and activation of astrocytes.

Collectively, our results suggested that Cur/SLNs-HU-211 reduced depressive behaviours in CORT-treated mice, alleviated DA/5-HT levels and increased GFAP and BDNF expression levels in the hippocampus and striatum. These effects are involved in the activation of MEK1/ERK1/2 signalling pathways. We also found that Rim had opposing effects in many experiments. Based on these results, we proposed a mechanism by which Cur/SLNsHU-211 acts to treat major depression. As shown in Fig. 11, this mechanism involved the MEK1/ERK1/2 signalling pathways. We demonstrated new curative properties of Cur/SLNs-

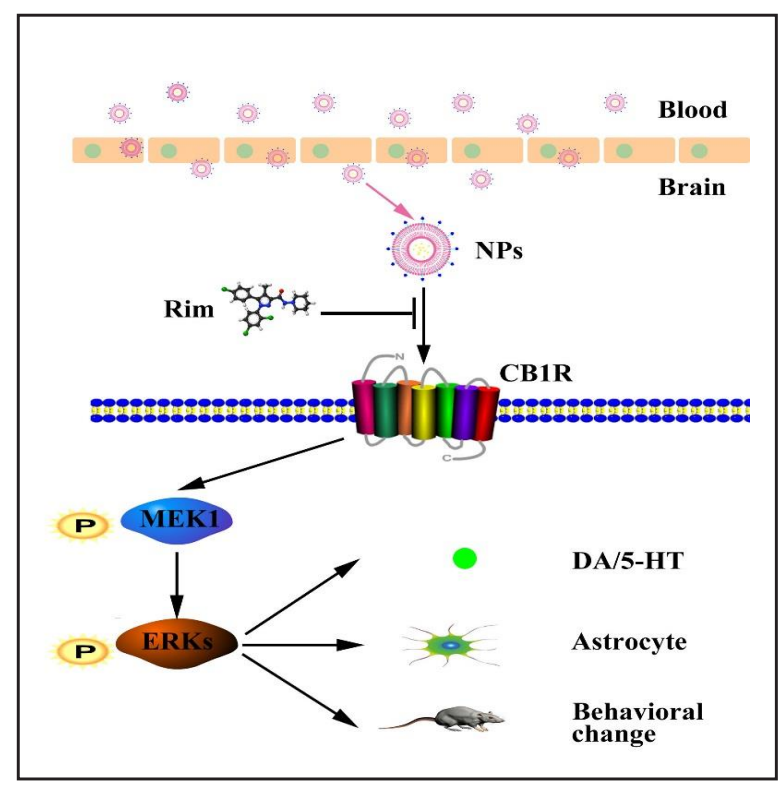

Fig. 11. Schematic representation of the mechanisms Cur/ SLNs-HU-211 reduced depressive behaviours in the CORTtreated mice through inhibition of neuronal cell death and involved in activation of MEK1/ERK1/2 signalling pathway. 
HU-211 for major depression as an auxiliary therapy and indicated a new therapeutic target CB1 in major depression. Further studies are needed to determine a more detailed mechanism and to develop a greater understanding of the therapeutic potency of Cur/SLNsHU-211.

\section{Conclusion}

Cur/SLNs-HU-211 nanoparticles were successfully prepared by a modified emulsification and low-temperature solidification method. In vitro and in vivo tests revealed that Cur/SLNs-HU-211 exerted antidepressant activity by targeting the endocannabinoid/ CB1 receptor system. Overall, the results of this study provided a compelling argument for the use of Cur/SLNs-HU-211 in the treatment of major depression.

\section{Acknowledgements}

This work was financially supported by the National Natural Science Foundation of China (Grant No. 31570849, 81671105), the National key research and development program (Grant No. 2016YFA0100800), and the Fundamental Research Funds for the Central Universities.

\section{Disclosure Statement}

No potential conflicts of interest are disclosed.

\section{References}

$>1$ Thornicroft G, Chatterji S, Evans-Lacko S, Gruber M, Sampson N, Aguilar-Gaxiola S, Al-Hamzawi A, Alonso J, Andrade L, Borges G, Bruffaerts R, Bunting B, Caldas de Almeida JM, Florescu S, de Girolamo G, Gureje O, Haro JM, He Y, Hinkov H, Karam E, Kawakami N, Lee S, Navarro-Mateu F, Piazza M, Posada-Villa J, Torres de Galvis Y, Kessler RC: Undertreatment of people with major depressive disorder in 21 countries. Br J Psychiatry 2016;10.1192/bjp.bp.116.188078

- Lang UE, Borgwardt S: Molecular Mechanisms of Depression: Perspectives on New Treatment Strategies. Cell Physiol Biochem 2013;31:761-777.

- 3 Li J, Wang P, Ying J, Chen Z, Yu S: Curcumin Attenuates Retinal Vascular Leakage by Inhibiting Calcium/ Calmodulin-Dependent Protein Kinase II Activity in Streptozotocin-Induced Diabetes. Cell Physiol Biochem 2016;39:1196-1208.

4 Zhou Y, Zhang T, Wang X, Wei X, Chen Y, Guo L, Zhang J, Wang C: Curcumin Modulates Macrophage Polarization Through the Inhibition of the Toll-Like Receptor 4 Expression and its Signaling Pathways. Cell Physiol Biochem 2015;36:631-641.

5 Morabito R, Falliti G, Geraci A, Spada GL, Marino A: Curcumin Protects -SH Groups and Sulphate Transport after Oxidative Damage in Human Erythrocytes. Cell Physiol Biochem 2015;36:345-357.

6 Fu XY, Zhang DW, Li YD, Zhao PW, Tang YQ, Niu JZ, Li Y: Curcumin treatment suppresses CCR7 expression and the differentiation and migration of human circulating fibrocytes. Cell Physiol Biochem 2015;35:489498.

7 Lopresti AL, Hood SD, Drummond PD: Multiple antidepressant potential modes of action of curcumin: a review of its anti-inflammatory, monoaminergic, antioxidant, immune-modulating and neuroprotective effects. J Psychopharmacol 2012;26:1512-1524.

-8 Yang W, Fu J, Yu M, Wang D, Rong Y, Yao P, Nussler AK, Yan H, Liu LG: Effects of Three Kinds of Curcuminoids on Anti-Oxidative System and Membrane Deformation of Human Peripheral Blood Erythrocytes in High Glucose Levels. Cell Physiol Biochem 2015;35:789-802.

-9 Kaufmann FN, Gazal M, Bastos CR, Kaster MP, Ghisleni G: Curcumin in depressive disorders: An overview of potential mechanisms, preclinical and clinical findings. Eur J Pharmacol 2016;784:192-198. 


\section{Cellular Physiology Cell Physiol Biochem 2017;42:2281-2294 \begin{tabular}{l|l} 
DOI: 10.1159/000480001 & $\begin{array}{l}\text { O 2017 The Author(s). Published by S. Karger AG, Basel } \\
\text { www.karger.com/cpb }\end{array}$ \\
\hline
\end{tabular}}

He et al.: Antidepressant Effects of Cur/SLNs-HU-211 Exerted by Targeting CB1R in Major Depression

10 Maas AI, Murray G, Henney H, 3rd, Kassem N, Legrand V, Mangelus M, Muizelaar JP, Stocchetti N, Knoller $\mathrm{N}$ : Efficacy and safety of dexanabinol in severe traumatic brain injury: results of a phase III randomised, placebo-controlled, clinical trial. Lancet Neurol 2006;5:38-45.

11 Naseri N, Valizadeh H, Zakeri-Milani P: Solid Lipid Nanoparticles and Nanostructured Lipid Carriers: Structure, Preparation and Application. Adv Pharm Bull 2015;5:305-313.

12 Jiang BP, Liu YM, Le L, Li ZY, Si JY, Liu XM, Chang Q, Pan RL: Cajaninstilbene Acid Prevents CorticosteroneInduced Apoptosis in PC12 Cells by Inhibiting the Mitochondrial Apoptotic Pathway. Cell Physiol Biochem 2014;34:1015-1026.

13 Gorzalka BB, Hill MN: Putative role of endocannabinoid signaling in the etiology of depression and actions of antidepressants. Prog Neuropsychopharmacol Biol Psychiatry 2011;35:1575-1585.

14 Onaivi ES: Cannabinoid receptors in brain: pharmacogenetics, neuropharmacology, neurotoxicology, and potential therapeutic applications. Int Rev Neurobiol 2009;88:335-369.

15 Zhang Z, Guo Y, Zhang S, Zhang Y, Wang Y, Ni W, Kong D, Chen W, Zheng S: Curcumin modulates cannabinoid receptors in liver fibrosis in vivo and inhibits extracellular matrix expression in hepatic stellate cells by suppressing cannabinoid receptor type-1 in vitro. Eur J Pharmacol 2013;721:133-140.

16 He X, Zhu Y, Wang M, Jing G, Zhu R, Wang S: Antidepressant effects of curcumin and HU-211 coencapsulated solid lipid nanoparticles against corticosterone-induced cellular and animal models of major depression. Int J Nanomedicine 2016;11:4975-4990.

17 Meye FJ, Trezza V, Vanderschuren LJ, Ramakers GM, Adan RA: Neutral antagonism at the cannabinoid 1 receptor: a safer treatment for obesity. Mol Psychiatry 2013;18:1294-1301.

18 Uchihara Y, Tanaka K, Asano T, Tamura F, Mizushima T: Superoxide dismutase overexpression protects against glucocorticoid-induced depressive-like behavioral phenotypes in mice. Biochem Biophys Res Commun 2016;469:873-877.

19 Kabir ZD, Lee AS, Burgdorf CE, Fischer D, Rajadhyaksha AM, Mok E, Rizzo B, Rice RC, Singh K, Ota KT, Gerhard DM, Schierberl KC, Glass M, Duman RS, Rajadhyaksha AM: Cacna1c in the Prefrontal Cortex Regulates Depression-Related Behaviors via REDD1. Neuropsychopharmacol 2016;10.1038/npp.2016.271

20 Olivares-Nazario M, Fernandez-Guasti A, Martinez-Mota L: Age-related changes in the antidepressant-like effect of desipramine and fluoxetine in the rat forced-swim test. Behav Pharmacol 2016;27:22-28.

-21 Chatterjee M, Verma P, Palit G: Comparative evaluation of Bacopa monniera and Panax quniquefolium in experimental anxiety and depressive models in mice. Indian J Exp Biol 2010;48:306-313.

-22 Ackermann TF, Kempe DS, Lang F, Lang UE: Hyperactivity and Enhanced Curiosity of Mice Expressing PKB/ SGK-resistant Glycogen Synthase Kinase-3 (GSK-3). Cell Physiol Biochem 2010;25:775-786.

23 Freitas AE, Egea J, Buendia I, Navarro E, Rada P, Cuadrado A, Rodrigues AL, Lopez MG: Agmatine induces Nrf2 and protects against corticosterone effects in hippocampal neuronal cell line. Mol Neurobiol 2015;51:1504-1519.

24 Xu W, Liu K, Lin QT, Ye XJ, Lu Y, Zhang XX, Zhao LC, Gao HC, Yan ZH: [Establishment and evaluation of a rat model of diabetes comorbid depression and its expression of glial fibrillary acidic protein in the brain]. Zhonghua Yi Xue Za Zhi 2016;96:2360-2364.

-25 Jiang B, Wang YJ, Wang H, Song L, Huang C, Zhu Q, Wu F, Zhang W: Antidepressant-like effects of fenofibrate in mice via the hippocampal BDNF signaling pathway. Br J Pharmacol 2016;10.1111/bph.13668

-26 Lee WD, Wang KC, Tsai YF, Chou PC, Tsai LK, Chien CL: Subarachnoid Hemorrhage Promotes Proliferation, Differentiation, and Migration of Neural Stem Cells via BDNF Upregulation. PLoS One 2016;11:e0165460.

-27 Wang GY, Fang H, Zhen YF, Xu G, Tian JL, Zhang YZ, Zhang DD, Zhang GY, Xu J, Zhang ZY, Qiu MY, Ma YJ, Zhang HR, Zhang XX: Sulforaphane Prevents Neuronal Apoptosis and Memory Impairment in Diabetic Rats. Cell Physiol Biochem 2016;39:901-907.

28 Caudal D, Alvarsson A, Bjorklund A, Svenningsson P: Depressive-like phenotype induced by AAV-mediated overexpression of human alpha-synuclein in midbrain dopaminergic neurons. Exp Neurol 2015;273:243252.

29 Kim YR, Kim HN, Hong KW, Shin HK, Choi BT: Anti-depressant effects of phosphodiesterase 3 inhibitor cilostazol in chronic mild stress-treated mice after ischemic stroke. Psychopharmacology (Berl) 2016;233:1055-1066. 